\title{
Evaluación y autoevaluación de los estudiantes mediante la gamificación y el uso de recursos tecnológicos
}

\author{
Daniel Palací-López ${ }^{\text {a }}$ Jesús Palacíb \\ ${ }^{a}$ Universitat Politècnica de València, dapalpe@gmail.com, ${ }^{\mathrm{b}}$ Universidad Pontificia de Comillas, \\ jpalaci@comillas.edu
}

\section{Resumen}

La reestructuración de los contenidos impartidos en las asignaturas que forman los actuales planes de estudio ha dificultado, en buena medida, la forma en que debería enfocarse el proceso de enseñanza-aprendizaje. A esto se suma el hecho de que muchos alumnos acceden a la universidad con un nivel de conocimientos inadecuado para el nivel requerido en diversas asignaturas.

El uso de herramientas eficientes para la evaluación de los conocimientos adquiridos por los estudiantes es, por tanto, de gran relevancia, ya que permite adaptar el nivel o el ritmo al que se imparte cada asignatura para mejorar la asimilación de la materia.

En esta línea se propone, mediante la gamificación, una actividad por equipos de no más de 8 alumnos en la que, haciendo uso de "Plickers", el profesorado adquiere información sobre el aprendizaje de los estudiantes a la vez que éstos puedan 'autoevaluarse' en tiempo real. También se plantea una actividad voluntaria, de carácter individual, evaluable tanto por el profesorado como por los compañeros de aula, cuya finalidad es analizar si se han adquirido las competencias básicas.

De este modo se propicia el trabajo equipo y el individual, posibilitando que tanto el profesor como los estudiantes revisan los conceptos trabajados y evalúan los resultados de aprendizaje adquiridos.

Palabras clave: resultados de aprendizaje, actividad grupal, plickers. 


\section{Introducción}

La metodología utilizada en el proceso de enseñanza-aprendizaje, en la Educación Superior, ha "sufrido" cambios constantes con la finalidad de paliar los efectos derivados de los cambios en los planes de estudio, que han hecho que en muchos casos los créditos asignados a las diferentes asignaturas sean insuficientes o, al menos, no de acorde al amplio temario de las mismas. A este hecho se ha sumado que la ubicación temporal de la materia y el nivel de conocimientos del alumnado no fueran de la mano. Aunque estos puntos débiles tienen un impacto a lo largo de todo el proceso, el trabajo que se presenta se centra en la evaluación del alumnado, en tanto en cuanto se entiende que se habrá alcanzado la optimización del proceso de enseñanza-aprendizaje si se ha conseguido que el alumnado asimile correctamente los contenidos de la materia y haya adquirido las competencias requeridas. La correcta y eficiente evaluación de dicha asimilación resulta, por tanto, indispensable. Para ello se propone hacer uso de la gamificación y de las Tecnología de la Información y Comunicación (TIC) en una actividad de carácter grupal, complementada con otra individual que requiere la implicación del resto de compañeros del aula, para poder evaluar si los objetivos perseguidos se han alcanzado.

La elección del uso de la gamificación y las TIC, para la primera actividad se sustenta en los buenos resultados que ha proporcionado tanto la primera (Bezanilla et al.,2014; Autor 1, Autor 2, y López, 2019) como por la segunda (Calvo, López, y Ruiz, 2017; Fuertes et al. 2016; López, Autor1, Autor 2,2015; Navarro y Olmo, 2014; Pintor et al. 2014). Por otra parte, la propuesta de la segunda actividad surge como una variante de la revisión por pares que tan buena acogida ha tenido en las metodologías utilizadas a lo largo de los últimos años (Monllor-Satoca et al., 2012) así como de los resultados más que positivos que se han obtenido al integrar al alumno en la evaluación (Morell et al. 2004).

Por otra parte, la asignatura donde se propone su uso es una de carácter cuantitativo (Estadística) del grado de Ingeniería Química, ofertado por la Universitat Politécnica de València (UPV). Más concretamente en el Tema 3, correspondiente al estudio de los conceptos básicos de cálculo de probabilidades.

\section{Propuesta}

En el presente epígrafe se expondrán las actividades mencionadas anteriormente, esto es la actividad de cierre (de carácter grupal) y la actividad posterior (de carácter individual). 


\subsection{Actividad de cierre}

La actividad de cierre tiene como objetivos fundamentales:

a) Propiciar el trabajo en grupo

b) Ser una herramienta de revisión y evaluación de los conceptos trabajados y los resultados de aprendizaje deseados, y para detectar puntos débiles que merezcan especial atención tanto por parte del profesorado como del alumnado de cara a futuras sesiones de aula, ya sea de teoría o de práctica, tutorías, etc.

Esta actividad requiere la formación de 3 equipos de 8 alumnos (partiendo de que el grupo está formado por 24 alumnos) que deberán "competir" entre ellos para obtener la mayor puntuación posible. Para ello se formulará a los alumnos una batería de preguntas sobre la materia impartida en la sesión teórica anterior y sobre los conceptos trabajados en las actividades previas durante la propia sesión. La actividad exige además el uso de la aplicación "Plickers", que se enmarca dentro de las denominadas Herramientas de Respuesta de Audiencia (HRA), pues se trata de un sistema de votación interactivo con múltiples utilidades, entre las que cabe destacar la realización del seguimiento de la asistencia al aula, evaluación de los conocimientos adquiridos en clases anteriores, y obtención de feedback inmediato a lo largo de las distintas sesiones. Además, el software necesario es gratuito, y no requiere que el alumno disponga de mandos, ordenador, móviles, ni ningún otro tipo de dispositivo para poder contestar a las cuestiones planteadas por el profesor.

Algunas recomendaciones de cara a la preparación previa de esta tarea son:

a) El docente debería organizar a los estudiantes en distintos grupos, y no permitir que sean los propios alumnos los que se organicen, ya que:

- Al elaborar dicha composición sería conveniente que los equipos fuesen lo más heterogéneos posibles para "romper" círculos cerrados que pudieran haberse formado a lo largo del primer semestre, y propiciar que éstos se "expandan".

- La composición de los grupos no debería hacerse pública hasta el comienzo de la actividad para evitar reparto de roles, intentos de cambios de componentes de un equipo por otros, etc.

b) Por las mismas razones que el punto anterior, tampoco debería hacerse público en qué consistirá la actividad hasta su comienzo; lo único que los estudiantes deberían saber es que deberán colaborar unos con otros y tener claros los conceptos trabajados durante la sesión, y que quizás ello influya en su nota más adelante...

Una vez formados los equipos, la actividad de cierre se llevará a cabo teniendo en cuenta el siguiente procedimiento: 
$\underline{\mathbf{1}}^{\mathbf{0}}$ : Cada equipo, compuesto por 8 alumnos, se distribuiría en 4 parejas que se sentarían una detrás de otra, tal y como se observa en la Figura 1 (para el caso particular en el que se consideran 3 equipos):

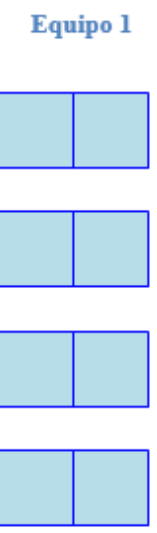

Equipo 2
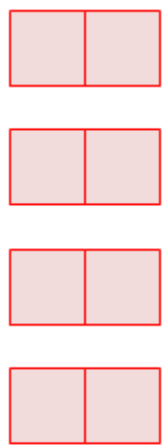

Equipo 3
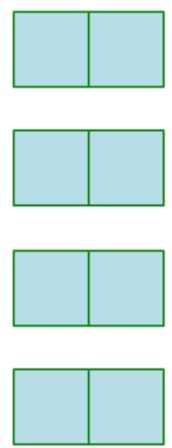

Fig. 1 Disposición integrantes equipos

吕: Se formularán un total de 12 o 24 preguntas (según tiempo disponible) de respuesta cerrada a todos los estudiantes, con tantas opciones de respuesta como parejas hay en cada grupo más una, al menos, por lo que el profesor deberá llevar las preguntas preparadas de antemano. Dichas preguntas se mostrarán en una presentación que se proyectará para que puedan leerlas todos los estudiantes sin problemas, además de escucharlas del propio docente. Tras cada pregunta (y sus correspondientes respuestas), se indicará también cuál era la respuesta correcta, a fin de que todo el mundo pueda llevar la cuenta del progreso de su equipo. Tanto la proyección de las preguntas como la recogida de las respuestas se llevaría a cabo mediante la aplicación "plickers".

$\underline{\mathbf{3}^{\text {o: }}}$ : Para asignar la puntuación a los equipos (se evalúa a todos los grupos de forma simultánea) se procederá de la siguiente manera:

3.1.- Una vez efectuada la $\mathbf{1}^{\mathbf{a}}$ pregunta, si la primera pareja de un determinado equipo la ha respondido correctamente, dicho equipo conseguirá 10 puntos; en caso contrario, si sí que lo ha hecho la segunda pareja, entonces el equipo conseguirá $\underline{8}$ puntos; si la primera pareja en contestar bien a la pregunta ha sido la tercera, el equipo anotará 6 puntos; y si ha sido la cuarta, entonces recibirán 4 puntos. $\mathrm{Si}$ ninguna pareja del equipo ha acertado la respuesta correcta, no recibirán ningún punto.

3.2.- Para la segunda pregunta el procedimiento es el mismo que antes, pero ahora la asignación de puntos sigue el siguiente esquema: si la segunda pareja ha respondido correctamente, su equipo anota 10 puntos; si ésta ha fallado, pero la tercera ha 
acertado, anotan $\underline{8 \text { puntos}}$; si no ha acertado ninguna de estas dos parejas, pero sí la $\underline{\text { cuarta }}$, entonces su equipo suma 6 puntos; si sólo ha acertado la primera pareja, les corresponderán 4 puntos.

3.4.- A partir de este punto el procedimiento es equivalente, pero con cada pregunta la pareja que da más puntos es la siguiente a la que daba el máximo número de puntos con la pregunta anterior, y la que antes daba 10 puntos ahora daría el mínimo, siguiendo el mismo orden hasta terminar las 12 o 24 preguntas que se harán en total a todo el grupo.

La Figura 2 muestra lo indicado para el caso particular en que se efectuaran 5 preguntas y se consideraran 3 equipos

\section{1a Pregunta 2a Pregunta $\quad \cdots \quad$ 5ạ Pregunta}

\section{0 puntos}

8 puntos

6 puntos
10 puntos

8 puntos

\section{0 puntos}

8 puntos

6 puntos

\section{4 puntos 6 puntos}

Fig. 2 Distribución de las puntuaciones

Por simplicidad es importante tener en cuenta que el número de respuestas posibles a cada pregunta debe ser superior al número de parejas en cada equipo, para evitar que cada pareja seleccione una opción y garantice así al menos 4 puntos cada vez. La comunicación entre parejas de cada equipo, durante el tiempo que tienen para contestar a cada pregunta (un minuto aproximadamente) está prohibida. Por otra parte, los datos identificativos de dicha actividad (título, tiempo estimado necesario para su puesta en marcha, materiales, instrucciones, ...) se recogen en la tabla 1. 
Tabla 1. Diseño de la actividad de cierre

\begin{tabular}{|l|}
\hline \multicolumn{1}{|c|}{ ACTIVIDAD DE CIERRE } \\
\hline Nombre: 'Cesta y puntos, todos a la vez' \\
\hline Duración estimada: 30 minutos \\
\hline Materiales: \\
- Tarjeta de "Plickers" \\
- Plantilla de anotaciones y puntuaciones (en 'Recursos', en PoliformaT) \\
- Formulario de la asignatura (impreso o en .pdf) \\
\hline Instrucciones: \\
Además de la explicación de la actividad, proporcionada antes de esta tabla: \\
- Cada estudiante debe responder a las cuestiones propuestas por el profesor haciendo uso de su \\
tarjeta de "plickers" en el tiempo establecido. \\
- Los alumnos pueden hacer anotaciones en la plantilla de anotaciones que se les proporcionará \\
antes de comenzar la actividad, o en el propio ordenador, descargada de la carpeta de 'Recursos' \\
de PoliformaT. Puede usar esta plantilla para llevar la cuenta de las puntuaciones de cada equipo \\
también. \\
-Los estudiantes pueden hacer uso a lo largo de esta actividad del formulario de la asignatura, \\
impreso o descargado desde PoliformaT. \\
\hline Evaluación: \\
- La evaluación en este caso se lleva a cabo en tiempo real durante la propia actividad, \\
recibiendo todos los estudiantes feedback inmediato de las respuestas correctas tras cada \\
pregunta (y la explicación, si conviene). Además, el profesor puede guardar las respuestas de \\
todos los alumnos (por parejas) a todas las preguntas planteadas, y usar esta información \\
para saber en qué cuestiones convendría incidir más o menos en futuras sesiones. \\
- En cuanto al posible impacto de esta actividad en la nota final de los estudiantes, se puede \\
ofrecer como incentivo a los estudiantes una mejora en la nota de su asignatura proporcional \\
a la puntuación obtenida por su equipo con respecto a la máxima posible, siempre que \\
superen una determinada puntuación. \\
\hline
\end{tabular}

Al tratarse de una actividad de cierre que cubre todos los conceptos impartidos relativos al Tema 3, los resultados de aprendizaje que se pretenden evaluar con esta actividad son todos los asociados a las competencias correspondientes (Autor1, Autor 2, 2019), además del trabajo en equipo y la gestión del tiempo (dado que deben responder "rápido" a las preguntas).

\subsection{Actividad posterior}

Tras la sesión de aula se propondrá a los estudiantes hacer una pequeña actividad de repaso voluntaria, con la finalidad de revisar los conceptos vistos en el aula, en la que el alumnado 
deberá plantear, en sus propias palabras, ejemplos similares a los vistos en cuanto a casuística que presentan. Los datos identificativos de dicha actividad se recogen en tabla 2

Tabla 2. Diseño de la actividad posterior

\begin{tabular}{|c|}
\hline ACTIVIDAD POSTERIOR \\
\hline Nombre: 'Crea tu paradoja personal' - Trabajo voluntario \\
\hline Duración estimada: 45 minutos \\
\hline $\begin{array}{l}\text { Materiales: } \\
\text { - Todos/cualquiera de los materiales usados o elaborados durante la sesión práctica } \\
\text { - Enunciado de la actividad posterior "Crea tu propia paradoja", en el apartado de 'Tareas' de } \\
\text { PoliformaT }\end{array}$ \\
\hline $\begin{array}{l}\text { Instrucciones: } \\
\text { Los estudiantes deben llevar a cabo esta tarea de manera individual. Se les pedirá: } \\
\text { - } \quad \text { Resumen de los problemas y paradojas presentados en clase: este ejercicio consistiría en la } \\
\text { presentación lo más concisa posible, pero precisa, del problema de Monty Hall } \\
\text { (https://www.youtube.com/watch?v=BzAhrFrnpGM) y las paradojas explicadas en la } \\
\text { sesión práctica, y su relación con los contenidos del Tema } 3 \text {. } \\
\text { Paradojas personales: el alumno tendría que buscar un ejemplo que se le ocurra, o que } \\
\text { haya leído en un libro o periódico, o visto en algún medio, etc. en que se dé la paradoja de } \\
\text { Simpson/Yule-Simpson (https://www.youtube.com/watch?v=ZDinnCwP3dg), y a ser } \\
\text { posible la de Ellsberg (https://es.wikipedia.org/wiki/Paradoja_de_Ellsberg), y justificar su } \\
\text { presencia en cada caso. A continuación, deberá preparar una presentación breve (menos de } \\
5 \text { diapositivas de PowerPoint, por ejemplo) de dicho/s ejemplo/s para presentar a la clase. }\end{array}$ \\
\hline $\begin{array}{l}\text { Evaluación: } \\
\text { - La evaluación en este caso se lleva a cabo mediante la corrección por parte del profesor de los } \\
\text { trabajos voluntarios presentados. Aquellos alumnos que hayan decidido realizarlos dispondrán a su } \\
\text { vez de dicha corrección. } \\
\text { - Además, el alumno o alumnos cuyo ejemplo mostrado en clase reciba la mejor valoración por parte } \\
\text { de sus compañeros tendrá una bonificación a la nota final (a discreción del profesor). }\end{array}$ \\
\hline
\end{tabular}

Los resultados de aprendizaje que se pretenden evaluar con esta actividad son:

- Sintetizar los conceptos aprendidos durante la sesión práctica relativa al Tema 3

- Analizar las consecuencias de las paradojas de Simpson/Yule-Simpson y su interpretación

- Definir o identificar casos en que se den las paradojas mencionadas, ya sea ficticios/imaginados o reales/observados. 


\section{Conclusiones}

La optimización del proceso de enseñanza-aprendizaje, en la Educación Superior, requiere una correcta asimilación, por parte del alumnado, de los contenidos de las diferentes materias que componen los planes de estudio. Así, en el trabajo que se presenta, se proponen dos actividades (de cierre y posterior) a desarrollar en la asignatura de Estadística del grado de Ingeniería Química, ofertado por la UPV, con la finalidad de evaluar si los estudiantes han alcanzado los objetivos propuestos. Aunque una de las actividades es grupal y la otra individual, la primera se desarrolla por completo dentro del aula y requiere del uso de la denominada gamificación y TIC, mientras que los resultados de la segunda se presentarán dentro del aula, y ambas actividades permiten tanto la evaluación por parte del profesor como la autoevaluación de los estudiantes. En trabajos posteriores se pretende evaluar el resultado de su implantación, así como la percepción que el alumnado ha tenido de las mismas.

\section{Agradecimientos}

Expresamos nuestro agradecimiento al Servei de Formació Permanent i Innovació Educativa así como a los Vicerrectorats d'Estudis de Grau i Política Lingüística i de Polítiques de Formació i Qualitat Educativa de la Universitat de València por la concesión del proyecto de innovación educativa UV-SFPIE_GER18-849069 que ha financiado parcialmente esta comunicación.

\section{Referencias}

Bezanilla, M.J., Arranz, S., Rayón, A., Rubio, I., Menchaca, I., Guenaga, M., Aguilar, E. (2014). Propuesta de evaluación en competencias genéricas mediante un juego serio. New Approaches in Educational Research. 3(1), pp. 44-54. doi: 10.7821/naer.3.3. 42-51.

López, M.I. y otros (2017).

Fuertes, A., García, M., Castaño, M.A., López, E., Zacares, M., Cobos, M., Ferris, R., Grimaldo, F. (2016). Uso de herramientas de respuesta de audiencia en la docencia presencial universitaria. Un primer contacto. En: Actas de las XXII Jenui. Almería, Spain, 261-268.

López, M.I., Autor 1, Autor 2 (2015).

Monllor-Satoca, D.; Guillén, E.; Lana-Villarreal, T.; Bonete, P.; Gómez, R. (2012). La evaluación por pares ("peer review") como método de enseñanza aprendizaje de la Química Física. En Tortosa, 
M. T.; Álvarez, J.D. y Pellín, N. (Eds.), Jornadas de Redes de Investigación en Docencia Universitaria X. Alicante

Morell Moll, T.; Aleson Carbonell, M. A.; Angulo Jerez, A.; Belda Medina, J. R.; Bellis, C.; Pérez Contreras, P. (2004). La integración de los alumnos en el proceso de evaluación.. En Bernabeu Pastor, G. y Sauleda Parés, N. (Eds.), Espacios de Investigación en la Profesionalización Docente Universitaria II. Alcoy: Marfil.

Navarro, J.M., Olmo, F. (2014). Socrative, una aplicación web 2.0 para evaluar la comprensión de los estudiantes. En Botti, V., Fernández, M.A., Simó, J. Fargueta, F. (eds.), Jornadas de Innovación Educativa y Docencia en Red de la Universitat Politécnica de València. (pp. 69-79). Valencia: Editorial Universitat Politécnica de València.

Autor 1, Autor 2, López, M.I. (2019).

Pintor Holguín, E., Gargantilla Madera, P., Herreros Ruiz-Valdepeñas, B., López del Hierro, M. (2014). Kahoot en docencia: una alternativa practica a los clickers. En: Actas de las XI Jornadas Internacionales de Innovación Universitaria. Educar para transformar. 322-329. 